\title{
Obligate and facultative paraneoplastic dermatoses: an overview
}

\author{
Stefano Caccavale ${ }^{1}$, Gabriella Brancaccio ${ }^{1}$, Marina Agozzino $^{1}$, Paola Vitiello ${ }^{1}$, Roberto Alfano ${ }^{2}$, \\ Giuseppe Argenziano ${ }^{1}$
}

1 Dermatology Unit, University of Campania Luigi Vanvitelli, Naples, Italy
2 Department of Anesthesiology, Surgery and Emergency, University of Campania Luigi Vanvitelli, Naples, Italy

Key words: dermatological paraneoplastic syndromes, obligate paraneoplastic dermatoses, facultative paraneoplastic dermatoses, malignancy, oncological dermatology

Citation: Caccavale S, Brancaccio G, Agozzino M, Vitiello P, Alfano R, Argenziano G. Obligate and facultative paraneoplastic dermatoses: an overview. Dermatol Pract Concept. 2018;8(3):191-197. DOI: https://doi.org/10.5826/dpc.0803a09

Received: January 15, 2018; Accepted: March 9, 2018; Published: July 31, 2018

Copyright: (02018 Caccavale et al. This is an open-access article distributed under the terms of the Creative Commons Attribution License, which permits unrestricted use, distribution, and reproduction in any medium, provided the original author and source are credited.

Funding: None.

Competing interests: The authors have no conflicts of interest to disclose.

All authors have contributed significantly to this publication.

Corresponding author: Stefano Caccavale, MD, Dermatology Unit, University of Campania Luigi Vanvitelli, Via Sergio Pansini, 5, 80131 Naples, Italy. Email: stefano85med@gmail.com
ABSTRACT Dermatological paraneoplastic syndromes are a group of cutaneous diseases associated with malig- nancy, but not directly related to the primary tumor itself or to its metastases. It is of utmost impor- tance for the dermatologist to recognize the major cutaneous paraneoplastic syndromes to diagnose the underlying tumors that trigger them as early as possible. In this overview, skin conditions that are highly correlated with malignancy, whose recognition implies a mandatory investigation of internal cancer, are described.

\section{Introduction}

The skin is the most accessible organ of the body and can be easily examined using noninvasive techniques, providing the doctor with clues that can be suggestive of systemic disturbances, thus contributing to the diagnosis of many diseases, including malignancies [1,2]. The skin may be directly or indirectly involved in malignancies. Direct involvement is due to the presence of tumor cells in the skin caused by direct tumor extension or metastasis. Indirect involvement of the skin can be determined by a variety of polypeptides, hormones, cytokines, antibodies, or growth factors related to the neoplasia. These inflammatory, proliferative, or metabolic factors act as mediators, interfering with cell communication and, consequently, with its activity. In this case, there is no presence of neoplastic cells in the skin, and this involvement is considered a dermatological paraneoplastic syndrome [1]. Thus, paraneoplastic syndromes are a group of diseases associated with a malignancy, but not directly related to the primary tumor itself or to its metastases.

It is not always easy to determine the correlation between a dermatologic finding and an internal neoplasm. The following criteria, defined by McLean in 1986 [3], should be verified to assess the causal relationship between the dermatosis and the potential underlying malignancy: (1) development of a dermatosis only after the development of a malignant 
TABLE 1. Association of Main Facultative Paraneoplastic Dermatoses with Systemic Neoplasms

Main Facultative Paraneoplastic Dermatoses

\begin{tabular}{|c|c|}
\hline Leser-Trélat sign & Gastrointestinal (GI), lymphoma, breast, lung \\
\hline Dermatomyositis & $\begin{array}{l}\text { Bronchogenic adenocarcinoma, ovary, genital, breast, cervix } \\
\text { carcinoma, GI }\end{array}$ \\
\hline Palmoplantar keratoderma & Esophageal carcinoma \\
\hline Pyoderma gangrenosum & Myelodysplastic syndrome, myeloma, leukemia, lymphoma \\
\hline Sweet syndrome & $\begin{array}{l}\text { Acute myelogenous leukemia, myelodysplastic syndrome, } \\
\text { myeloproliferative disorders, lymphoproliferative disorders }\end{array}$ \\
\hline Trousseau's syndrome & Pancreas, lung, stomach \\
\hline Vasculitis & Leukemia, lymphoma, lung, multiple myeloma \\
\hline Pruritus & Hodgkin and non-Hodgkin lymphoma \\
\hline Extramammary Paget's disease & GI, genitourinary (GU) \\
\hline Digital clubbing & Lung, mesothelioma \\
\hline Raynaud's phenomenon & Testicle, GU, GI \\
\hline Multicentric reticulohistiocytosis & Hematologic malignancies, breast, stomach \\
\hline Amyloidosis & Multiple myeloma, Hodgkin lymphoma, kidney \\
\hline Flushing & Carcinoid, medullary carcinoma of thyroid, leukemia, kidney \\
\hline Linear IgA dermatosis & $\begin{array}{l}\text { Lymphoproliferative malignancies, esophagus, renal cell, thyroid, } \\
\text { bladder }\end{array}$ \\
\hline Mucous membrane pemphigoid & $\begin{array}{l}\text { Chronic lymphocytic leukemia, non-Hodgkin lymphoma, acute } \\
\text { myeloid leukemia, pancreas, lung, ovary, cervix, liver, GI, thymoma }\end{array}$ \\
\hline Bullous pemphigoid & $\begin{array}{l}\text { Breast, prostate, lung, thyroid, larynx, GI, cervix, uterus, bladder, } \\
\text { lymphoreticular system, kidney, melanoma, and squamous cell } \\
\text { carcinomas }\end{array}$ \\
\hline
\end{tabular}

neoplasm and (2) both the dermatosis and the malignant neoplasm follow a parallel clinical course, so the cutaneous symptoms disappear when the tumor is treated and reappear in cases of recurrence or metastasis [3].

Paraneoplastic syndromes occur in about $7 \%$ to $15 \%$ of patients with cancer and may be the presenting sign of an unknown neoplasm, precede the diagnosis of malignancy, occur late in the course of illness, or be the first sign of recurrence. Their recognition may result in earlier diagnosis and better prognosis for the patient, prolonging life expectancy [4-8].

A few rare paraneoplastic dermatoses are consistently associated with malignancies in almost $100 \%$ of the cases (obligate paraneoplastic dermatoses) [1-29]; others are more common skin disorders, associated with tumors in only $3 \%$ to $30 \%$ of the cases, so the coexistence of these dermatoses and neoplasms may be coincidental and the causal link is more controversial (facultative paraneoplastic dermatoses) [9,10,30] (Table 1).

In this overview, dermatoses that highly correlate with malignancy and that, when recognized, require mandatory investigation of internal cancer-excluding those disorders showing a genetic pattern (genodermatoses)—are described [1].

\section{Acanthosis Nigricans Maligna}

Acanthosis nigricans (AN) can be classified as benign or malignant [1]. Benign AN is relatively common; it may be familial or occur in association with drug use, obesity, and/or endocrinopathy, thus being regarded as a sign of hyperinsulinism and insulin resistance. It is characterized by the presence of hyperpigmented, velvety plaques most often seen on the sides and nape of the neck ("dirty neck" appearance) and other flexural (antecubital, popliteal fossae) or intertriginous areas (inguinal, axillae, inframammary folds). Acrochordons frequently arise on the plaques of AN [11].

By contrast, acanthosis nigricans maligna (ANM) can be distinguished by its severity and its rapid and extensive spread. Sudden-onset AN is often associated with malignancy, especially in adult (average age 40 years) nonobese patients [1]. ANM may be more widespread or generalized and involve atypical areas (interdigital, knuckles, soles, palmar, eyelids, perioral) and even mucosal surfaces, including 


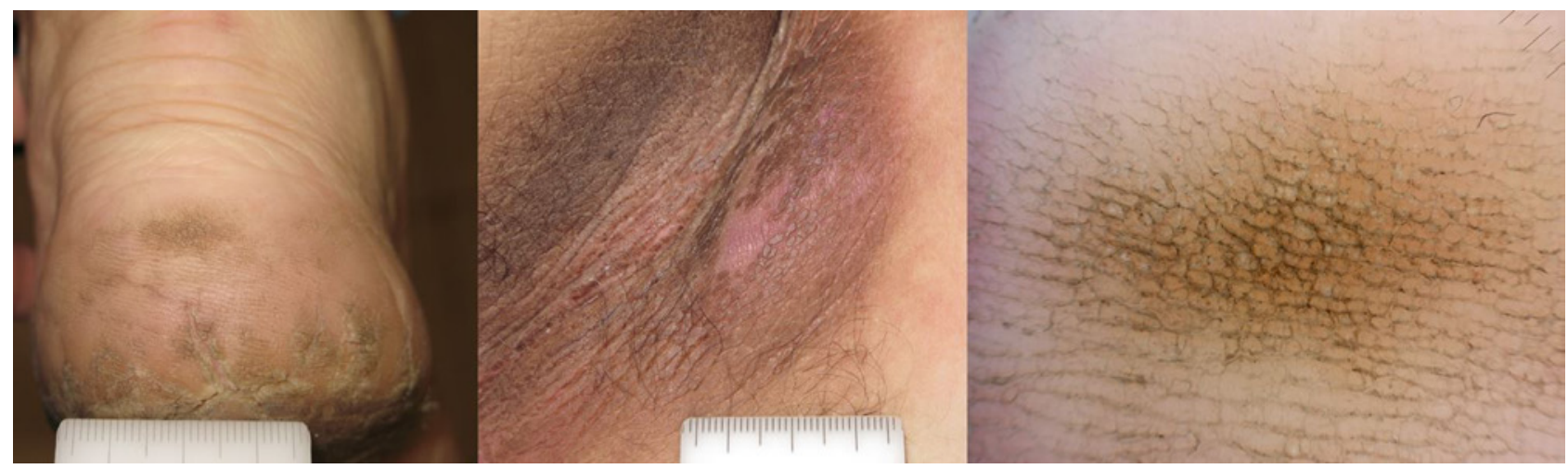

Figure 1. Acanthosis nigricans maligna: clinical and dermatoscopical pictures. [Copyright: @2018 Caccavale et al.]

the palate. Palmar and plantar keratoderma with prominent fingerprint markings known as pachydermatoglyphy or tripe palms may be seen $[10,11]$ (Figure 1). Skin biopsy is rarely necessary. Common histopathologic findings are hyperkeratosis, papillomatosis, acanthosis alongside epidermal atrophy, and absence of an inflammatory infiltrate.

Nearly all malignancies associated with ANM are adenocarcinomas (most commonly gastrointestinal adenocarcinomas). Many others have been reported, including adrenal, bile duct, bladder, breast, cervical, endometrium, liver, larynx, lung, lymphoid hematological malignancies, ovary, pituitary, prostate, kidney, testicle, thyroid, and sarcomas [11]. ANM can precede or occur simultaneously or after the diagnosis of cancer [1]. In a review study, this dermatological finding was observed in $58 \%$ of patients before tumor diagnosis $[1,12]$.

The pathogenesis for the development of ANM is debated (insulin-like growth factors or transforming growth factor alpha (TGF- $\alpha$ ) may be responsible for the epidermal proliferation) [10].

Depending on the clinical scenario, an extensive search for visceral malignancy may be warranted (fecal occult blood testing, endoscopy, imaging, etc.). In the case of ANM, as for all paraneoplastic dermatoses, skin lesions may regress after systemic chemotherapy or radical surgery.

\section{Acrokeratosis Paraneoplastica (Bazex Syndrome)}

Patients affected by acrokeratosis paraneoplastica are typically men older than 40 years [11]. Cutaneous lesions are psoriasiform and manifest as asymptomatic, symmetrical erythematous-violaceous scaly patches. However, their distribution is not typical of psoriasis: in initial stages, the dermatosis involves the bridge of the nose, auricular helices, and distal ends of the extremities. As the disease progresses, desquamation may affect the dorsal and palmoplantar regions, producing a violaceous keratoderma. In advanced stages, other areas may be affected such as the elbows, knees, legs, arms, cheeks, and scalp, with centripetal distribution of the lesions. Nails may also be involved with subungual hyperkeratosis, onycholysis, and dystrophy. Bullous lesions, mainly in the hands and feet, have been described [1].

Biopsies are nonspecific. Histologically, some psoriasiform features are present including hyperkeratosis, parakeratosis, and a superficial lymphohistiocytic infiltrate, but other non-psoriasiform changes also exist (vacuolar degeneration with melanin-containing macrophages in the dermis and dyskeratotic keratinocytes) [11].

Almost all the cases cited in the literature were associated with malignancy. This syndrome typically precedes the diagnosis of malignancy by approximately 2 to 6 months in $65 \%$ to $70 \%$ of patients. About $80 \%$ of cases are associated with tumors of the upper aerodigestive tract (oral cavity, larynx, pharynx, trachea, esophagus, and lung), commonly squamous cell carcinomas (SCC) or metastasis to cervical lymph nodes. In a retrospective study, $49 \%$ of cancers involved the oropharynx and larynx, followed by the lung $(17 \%)$ and esophagus $(10.6 \%)$ [1]. Additional isolated cases associated with breast cancer, cholangiocarcinoma, colon adenocarcinoma, and Hodgkin lymphoma have been reported [1]. Case reports also describe SCCs of the thymus, vulva, and skin $[13,14]$.

Its underlying mechanism is not well understood. Immunological factors with antibodies directed against the tumor in a cross-reaction with the epidermis or basement membrane have been considered. Possibly, a tumor production of a keratinocyte growth factor such as TGF- $\alpha$ may be involved [1].

Paraneoplastic acrokeratosis generally responds to successful treatment of the underlying tumor, and fails to improve when the neoplasm persists. Topical corticosteroids and systemic retinoids may be helpful.

The physician should inquire regarding risk factors for malignancy (smoking habits, alcohol consumption, weight loss, family history) and perform a complete physical examination, including head and neck and endoscopic and pelvic examination in women $[13,14]$. 

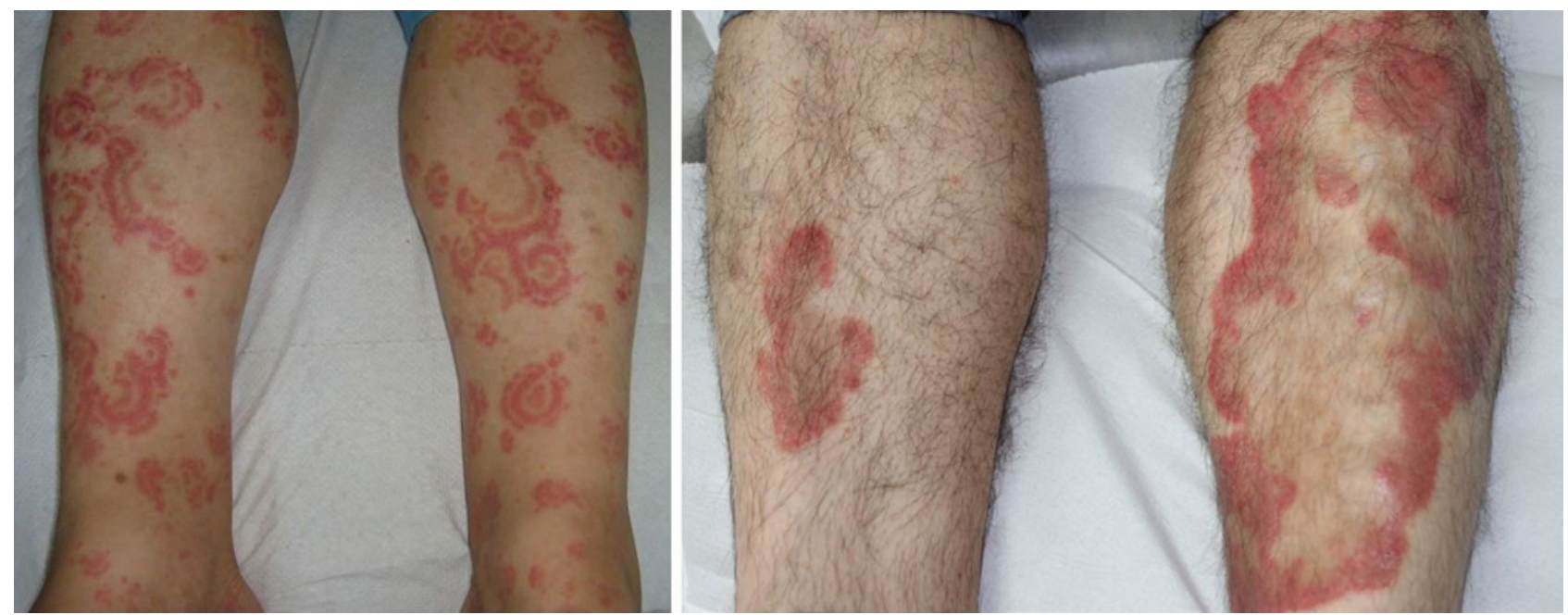

Figure 2. Erythema gyratum repens (left) and erythema annulare centrifugum (right). [Copyright: (O2018 Caccavale et al.]

\section{Erythema Gyratum Repens}

Erythema gyratum repens (gyrate from the Greek, meaning “a circle"; repens from the Latin, meaning "to creep") (EGR) is a rare paraneoplastic dermatosis, usually occurring in patients older than 40 years, with a mean age of about 60 years; the male-to-female ratio is $2: 1$ [1,11,15].

The primary lesion is a pruriginous, macular erythema. Numerous serpiginous bands are arranged in a parallel configuration of red swirls over most of the body, producing concentric figures that resemble a wood surface ("wood-grained" appearance) (Figure 2). The edges of the lesions migrate at a rapid rate, about $1 \mathrm{~cm} /$ day. A slight scale can be found along the trailing edge of erythema. The hands, feet, and face are often spared $[1,15]$. Peripheral eosinophilia is frequent.

The presentation of EGR is so typical that a differential diagnosis is difficult to generate. Another gyrate erythema, erythema annulare centrifugum (EAC), demonstrates polycyclic erythematous rings with a trailing edge of scale. Similarly, this eruption can be pruritic. Unlike EGR, EAC migrates slowly and is usually localized to smaller areas on the trunk and extremities [11,16] (Figure 2). Other figurate erythemas are erythema chronicum migrans and erythema marginatum.

Patients with EGR should be mandatorily evaluated for the presence of malignancy because the disease is virtually always associated with an internal cancer [1]. Malignant neoplasms are found in $82 \%$ of patients with EGR. Lung cancer is the most common $(32 \%)$, followed by cancer of the esophagus $(8 \%)$ and breast $(6 \%)$. Other malignancies have been associated, such as colon, stomach, bladder, prostate, uterine, rectal, and pancreatic cancer and multiple myeloma. The diagnosis of EGR precedes the diagnosis of the neoplasia in approximately $80 \%$ of patients, on average from 4 to 9 months [13].
In patients with EGR who did not have an underlying internal cancer, nonneoplastic conditions including tuberculosis, pregnancy, calcinosis, esophageal dysmotility, sclerodactyly, Sjögren's syndrome, and CREST syndrome may be rarely associated $[1,17,18]$. Histopathology is nonspecific, showing mild hyperkeratosis, parakeratosis, acanthosis, and spongiosis with a perivascular mononuclear inflammatory infiltrate in the dermis [1]. Its pathophysiology remains unknown. Immune mechanisms are probably involved since immunosuppression accompanies the resolution of EGR [1]. In paraneoplastic cases, therapy is aimed at treating the underlying malignancy. Systemic corticosteroids have been tried with only partial success.

\section{Necrolytic Migratory Erythema or Glucagonoma Syndrome}

Necrolytic migratory erythema (NME) is more common in women more than 45 years of age, with an average age of onset of 52 years [1]. NME presents as a pruritic and sometimes painful mucocutaneous eruption with a pinkish, maculopapular erythema with irregular edges and annular or arciform lesions that tend to coalesce and adopt a polycyclic pattern. Lesions are prominent in areas of pressure or trauma, commonly in intertriginous areas, groin, perineum, buttocks, distal extremities, and the central face. Sometimes there is formation of flaccid blisters that rupture easily, forming erosions and crusts, while new vesicles continue to develop along the edges. Residual hyperpigmentation at sites previously affected is common [1,19]. The disease has a waxing and waning course. The eruption may resemble such dermatoses as pemphigus foliaceus, acrodermatitis enteropathica, chronic mucocutaneous candidiasis, psoriasis, and severe seborrheic dermatitis [11]. 
NME is typically associated with glucagonoma syndrome. Glucagonoma is a rare endocrine, usually slow-growing, tumor of pancreatic alpha cells. Glucagon-secreting tumors of the pancreas are responsible of the triad hyperglucagonemia, whose levels are greater than $1000 \mathrm{pg} / \mathrm{mL}$, diabetes or glucose intolerance and NME [1,19]. Other common manifestations are glossitis, angular cheilitis, weight loss, diarrhea, steatorrhea, abdominal pain, normocytic anemia, hypoaminoacidemia, thromboembolic disease, and psychiatric disturbances $[1,19]$. NME can be associated also with non-glucagon-secreting tumors (small-cell lung cancer, liver cancer, insulin-secreting tumors, and duodenal neoplasms), and in malabsorption syndromes, liver failure, inflammatory bowel disease, and celiac disease, leading to pseudoglucagonoma syndrome [1].

A CT scan may be useful in the diagnosis. About $95 \%$ of glucagonomas are positive in somatostatin receptor scintigraphy. Somatostatin positivity may be useful in the treatment of the symptoms and signs of glucagonoma (octreotide, a somatostatin analogue, inhibits glucagon production) [1]. Celiac arteriography is considered most sensitive to locate the tumor because of its vascularity [10].

Histological findings of NME are nonspecific (edema and epidermal acanthosis with basal cell hyperplasia, spongiosis, parakeratosis with vacuolated epidermal cells associated with necrosis of the upper epidermis, cleft-like detachment from the deeper epidermis and loss of the granular cell layer, mild to moderate perivascular inflammatory infiltrate) $[1,10]$. The pathogenesis is poorly understood. Various metabolic abnormalities and nutritional deficiencies have been implicated, including hypoaminoacidemia, zinc, and essential fatty acid deficiencies [10].

\section{Acquired Hypertrichosis Lanuginosa}

Acquired hypertrichosis lanuginosa (AHL) is a rare paraneoplastic condition characterized by the sudden appearance of long, thin, soft, unpigmented, lanugo-like hair initially on the face and ears and then on the trunk, axillae, and extremities, with sparing of the palms, soles, and genital area [2]. It is more common in women than in men. Other symptoms are painful glossitis, angular cheilitis, and papillary hypertrophy of the tongue $[2,20]$.

In men, AHL is most commonly associated with lung cancer, followed by colorectal cancer. In women, the most common neoplasms are, in order of frequency, colorectal cancer, lung cancer, and breast cancer. However, AHL has also been described in association with other cancers (ovary, uterus, bladder, pancreas, kidney, lymphomas, and leukemia) [2,20].

AHL must be differentiated from hypertrichosis associated with endocrine or metabolic alterations and use of medication (cyclosporine, penicillamine, glucocorticoids, interferon, minoxidil, phenytoin, spironolactone, and cetuximab) $[1,20]$. An extensive clinical history and physical examination are necessary, in conjunction with blood tests, laboratory screening, and imaging (chest radiography, CT, colonoscopy, and, in women, mammography) [1]. Pathogenesis of AHL is unclear. Prolongation of the anagen phase of vellus hairs by a tumor-induced serum growth factor has been hypothesized $[1,10]$.

\section{Ichthyosis Acquisita}

Ichthyosis acquisita is a cutaneous keratinization disorder, characterized by small white or brownish rhomboidal scales that rise above the skin surface, particularly localized symmetrically on the extensor surfaces of the extremities and on the trunk and scalp. The dry scales may be also thickened and widespread [13]. Flexures, palms, and soles are spared [21].

Ichthyosis may be due to systemic diseases (eg, leprosy, hypothyroidism, lymphoma, and AIDS) or to drug intake (eg, nicotinic acid, triparanol, and butyrophenones) [13]. New onset of ichthyosis in adult life is often related to an underlying malignancy [13,21]. Ichthyosis acquisita is mostly seen in association with Hodgkin lymphoma, non-Hodgkin lymphoma (including mycosis fungoides), other lymphoproliferative diseases (eg, reticulolymphosarcoma, multiple myeloma), and nonlymphoproliferative diseases (Kaposi sarcoma, leiomyosarcoma, breast, ovarian, lung, liver, and cervical carcinomas) [21,22]. Acquired ichthyosis usually appears several weeks or months after detection of the cancer [2].

It has been suggested that TGF- $\alpha$ secreted from the tumor cells, may be implicated in the pathogenesis [22]. Treatment of ichthyosis includes removal of exacerbating factors and of related systemic diseases, as well as applying moisturizers and keratolytics [13].

\section{Pityriasis Rotunda}

Patients often manifest pityriasis rotunda (PR) between 20 and 45 years of age (range of 2 to 89) [23], except among the Sardinian cohort, in which patients presented during childhood [24]. Incidence is equal among men and women. The disease lasts from several months to more than 20 years, with reports of exacerbation during winter months [25]. PR is a rare disease characterized by round or oval, well-defined, scaly, hypo- or hyperpigmented patches or thin plaques typically found on the trunk, back, buttocks, or arms, but that can occur in every area of the body. Lesions number ranges in number from 1 to over 100, and the diameter from 1-10 cm. The hands, feet, and face are usually spared. Lesions are asymptomatic but may be associated with minimal pruritus [13]. 
TABLE 2. Diagnostic Criteria for Paraneoplastic Pemphigus (proposed by Camisa and Helm) [29]

Major Criteria

\begin{tabular}{|l|l|}
\hline Polymorphous skin eruption & Histological evidence of intraepithelial acantholysis \\
\hline Concurrent internal neoplasia & $\begin{array}{l}\text { Positive direct immunofluorescence (DIF) with deposits } \\
\text { both intercellularly and at the basement membrane zone } \\
\text { with IgG and C3 deposition }\end{array}$ \\
\hline Antibodies with an immunoprecipitation specific pattern & $\begin{array}{l}\text { Positive indirect immunofluorescence (IIF) on rat bladder } \\
\text { epithelium }\end{array}$ \\
\hline
\end{tabular}

One-third of patients have an underlying disease, including tuberculosis, leprosy, and liver, kidney, heart, and lung diseases. Associated neoplasms include hepatocellular, gastric and esophageal carcinoma, prostate cancer, chronic lymphocytic leukemia, and multiple myeloma [1].

PR has been classified into 2 distinct subtypes. Type 1 occurs predominantly in elderly patients of Asian and African descent and is frequently associated with systemic illness, infections, or malignancy. Lesions are often hyperpigmented and generally fewer than 40. Type 1 PR often improves with treatment of underlying systemic illness [26]. Type 2 occurs in younger patients of Northern European descent. It has a strong hereditary predisposition and it is believed to belong to the spectrum of congenital ichthyoses. Lesions are typically hypopigmented and more numerous. Type 2 PR is often self-limiting and improves through adulthood [26]. PR can be treated with retinoids, salicylates, or lactate emollients.

\section{Paraneoplastic Pemphigus}

Paraneoplastic pemphigus (PNP) is a severe acantholytic mucocutaneous syndrome characterized by painful mucosal erosions, ulcerations, and polymorphous skin lesions that progress to blistering eruptions on the trunk and extremities $[27,28]$. Oral involvement with persistent, painful stomatitis is seen in almost all cases and can often be the first symptom. Oral lesions may be severe, and affect the entire mouth, with diffuse areas of shallow ulceration, and lips, with hemorrhagic crusting, and also the hypopharynx and esophagus; they may also involve other mucosa (conjunctival and anorectal) with ulcers and erosions. Contrary to pemphigus vulgaris, acral and paronychial involvement is common in PNP. Cutaneous manifestations can be classified in several groups: (1) pemphigus-like, (2) bullous pemphigoid-like, (3) erythema multiforme-like, (4) graft-versus-host disease, and (5) lichen planus-like [27,28].

The prognosis of PNP is severe. Some patients have respiratory complications, such as bronchiolitis obliterans, responsible for dyspnea and respiratory failure. The high mortality rate of PNP (75\%-80\%) is also secondary to sepsis and bleed- ing [1]. The diagnostic criteria for PNP can be distinguished into major criteria and minor criteria (Table 2) [29]: 3 major criteria or 2 major and 2 minor are needed [1,11]. Contrary to pemphigus vulgaris, in which direct immunofluorescence shows only intercellular deposition in epithelial cells, both basement membrane and epidermis are affected in PNP [1].

Two-thirds of patients have a recognized neoplasia at the onset of PNP. Approximately, $80 \%$ of associated malignancies are of hematological origin (non-Hodgkin B-cell lymphoma, chronic lymphocytic leukemia, Hodgkin lymphoma, T-cell lymphoma, Castleman disease, thymoma, Waldenström's macroglobulinemia). In children and adolescents, association with Castleman disease is the most frequent [1]. Other neoplasms associated with PNP include Kaposi's sarcoma and carcinomas of the breast, skin, mucous membranes, lung, uterus, ovary, stomach, liver, and gastrointestinal tract [13].

PNP is characterized by production of autoantibodies directed against proteins of the plakin and cadherin families involved in cell architecture maintenance and tissue cohesion. Autoantibodies immunoprecipitate keratinocyte antigens at $250 \mathrm{kD}$ (desmoplakin I), $230 \mathrm{kD}$ [bullous pemphigoid antigen I (BPAG1)], $210 \mathrm{kD}$ (desmoplakin II and envoplakin), and $190 \mathrm{kD}$ (periplakin) in all samples and at $170 \mathrm{kD}$ in some samples. The production of autoantibodies that bind epidermal proteins is responsible of skin and mucosal displacement $[13,27,28]$.

\section{Conclusions}

Skin, being the most visible and external organ of the body, may be considered as a mirror for many systemic diseases [7]. It is of utmost importance for the dermatologist to recognize the major cutaneous paraneoplastic syndromes to diagnose the underlying tumors that trigger them as early as possible.

\section{References}

1. Silva JA, Mesquita KC, Igreja AC, et al. Paraneoplastic cutaneous manifestations: concepts and updates. An Bras Dermatol. 2013;88(1):9-22. doi: 10.1590/S0365-05962013000100001. 
2. Yuste Chaves M, Unamuno Pérez P. Cutaneous manifestations of systemic malignancies: part 2. Actas Dermosifiliogr. 2013;104(7):543-553. doi: 10.1016/j.adengl.2012.05.026.

3. McLean DI. Cutaneous paraneoplastic syndromes. Arch Dermatol. 1986;122(7):765-767. doi: 10.1001/archderm.1986. 01660190043013.

4. Brenner S, Tamir E, Maharshak N, Shapira J. Cutaneous manifestations of internal malignancies. Clin Dermatol. 2001;19(3):290297. doi: 10.1016/S0738-081X(01)00174-2.

5. Moore RL, Devere TS. Epidermal manifestations of internal malignancy. Dermatol Clin. 2008;26(1):17-29, vii. doi: 10.1016/j. det.2007.08.008.

6. Pipkin CA, Lio PA. Cutaneous manifestations of internal malignancies: an overview. Dermatol Clin. 2008;26(1):1-15, vii. doi: 10.1016/j.det.2007.08.002.

7. Vora RV, Kota RS, Diwan NG, Jivani NB, Gandhi SS. Skin: A mirror of internal malignancy. Indian J Med Paediatr Oncol. 2016;37(4):214-222. doi: 10.4103/0971-5851.195730.

8. Miyashiro D, Sanches JA. Paraneoplastic skin disorders: a review. G Ital Dermatol Venereol. 2016;151(1):55-76.

9. Károlyi Z. [Paraneoplastic dermatoses]. Orv Hetil. 2002;143(31): 1827-1833.

10. Thomas I, Schwartz RA. Cutaneous paraneoplastic syndromes: uncommon presentations. Clin Dermatol. 2005;23(6):593-600. doi: 10.1016/j.clindermatol.2005.01.006.

11. Stone SP, Buescher LS. Life-threatening paraneoplastic cutaneous syndromes. Clin Dermatol. 2005;23(3):301-306. doi: 10.1016/j. clindermatol.2004.06.011.

12. Ehst BD, Minzer-Conzetti K, Swerdlin A, Devere TS. Cutaneous manifestations of internal malignancy. Curr Probl Surg. 2010;47(5):384-445. doi: 10.1067/j.cpsurg.2010.01.003.

13. Abreu Velez AM, Howard MS. Diagnosis and treatment of cutaneous paraneoplastic disorders. Dermatol Ther. 2010;23(6):662675. doi: 10.1111/j.1529-8019.2010.01371.x.

14. Räßler F, Goetze S, Elsner P. Acrokeratosis paraneoplastica (Bazex syndrome) - a systematic review on risk factors, diagnosis, prognosis and management. J Eur Acad Dermatol Venereol. 2017;31(7):1119-1136. doi: 10.1111/jdv.14199.

15. Boyd AS, Neldner KH, Menter A. Erythema gyratum repens: a paraneoplastic eruption. J Am Acad Dermatol. 1992;26(5 Pt 1):757762. doi: 10.1016/0190-9622(92)70107-Q.

16. Tyring SK. Reactive erythemas: erythema annulare centrifugum and erythema gyratum repens. Clin Dermatol. 1993;11(1):135139. doi: 10.1016/0738-081X(93)90110-X.

17. Lo Schiavo A, Caccavale S, Orlando I, Tirri R. Erythema gyratum repens and rheumatoid arthritis: an unrecognized association? Indian J Dermatol Venereol Leprol. 2012;78(1):122. doi: 10.4103/0378-6323.90974.
18. Rongioletti F, Fausti V, Parodi A. Erythema gyratum repens is not an obligate paraneoplastic disease: a systematic review of the literature and personal experience. J Eur Acad Dermatol Venereol. 2014;28(1):112-115. doi: 10.1111/j.1468-3083.2012.04663.x

19. John AM, Schwartz RA. Glucagonoma syndrome: a review and update on treatment. J Eur Acad Dermatol Venereol. 2016;30(12):2016-2022. doi: 10.1111/jdv.13752

20. Slee PH, van der Waal RI, Schagen van Leeuwen JH, et al. Paraneoplastic hypertrichosis lanuginosa acquisita: uncommon or overlooked? Br J Dermatol. 2007;157(6):1087-1092. doi: 10.1111/j.1365-2133.2007.08253.x

21. Patel N, Spencer LA, English JC III, Zirwas MJ. Acquired ichthyosis. J Am Acad Dermatol. 2006;55(4):647-656. doi: 10.1016/j. jaad.2006.04.047

22. Kleyn CE, Lai-Cheong JE, Bell HK. Cutaneous manifestations of internal malignancy: diagnosis and management. Am J Clin Dermatol. 2006;7(2):71-84. doi: 10.2165/00128071-20060702000001

23. Friedmann AC, Ameen M, Swale VJ. Familial pityriasis rotunda in black-skinned patients; a first report. $\mathrm{Br} J$ Dermatol. 2007;156(6):1365-1367. doi: 10.1111/j.1365-2133.2007. 07874.x

24. Aste N, Pau M, Aste N, Biggio P. Pityriasis rotunda: a survey of 42 cases observed in Sardinia, Italy. Dermatology. 1997;194(1):32-35. doi: 10.1159/000246053

25. Batra P, Cheung W, Meehan SA, Pomeranz M. Pityriasis rotunda. Dermatol Online J. 2009;15(8):14.

26. Grimalt R, Gelmetti C, Brusasco A, Tadini G, Caputo R. Pityriasis rotunda: report of a familial occurrence and review of the literature. J Am Acad Dermatol. 1994;31(5 Pt 2):866-871. doi: 10.1016/S0190-9622(94)70248-9

27. Kartan S, Shi VY, Clark AK, Chan LS. Paraneoplastic pemphigus and autoimmune blistering diseases associated with neoplasm: characteristics, diagnosis, associated neoplasms, proposed pathogenesis, treatment. Am J Clin Dermatol.2017;18(1):105-126. doi: 10.1007/s40257-016-0235-z

28. Wieczorek M, Czernik A. Paraneoplastic pemphigus: a short review. Clin Cosmet Investig Dermatol. 2016;9:291-295. doi: 10.2147/CCID.S100802

29. Camisa C, Helm TN. Paraneoplastic pemphigus is a distinct neoplasia-induced autoimmune disease. Arch Dermatol. 1993;129(7):883-886. doi: 10.1001/archderm.1993. 01680280071014

30. Caccavale S. The association of bullous pemphigoid and malignancy: a case control study. G Ital Dermatol Venereol. 2015;150(6):764-765. 